\title{
Non-commutative Donaldson-Thomas invariants and the conifold
}

\author{
BALÁZS SZENDRŐI
}

\begin{abstract}
Given a quiver algebra $A$ with relations defined by a superpotential, this paper defines a set of invariants of $A$ counting framed cyclic $A$-modules, analogous to rank-1 Donaldson-Thomas invariants of Calabi-Yau threefolds. For the special case when $A$ is the non-commutative crepant resolution of the threefold ordinary double point, it is proved using torus localization that the invariants count certain pyramid-shaped partition-like configurations, or equivalently infinite dimer configurations in the square dimer model with a fixed boundary condition. The resulting partition function admits an infinite product expansion, which factorizes into the rank-1 Donaldson-Thomas partition functions of the commutative crepant resolution of the singularity and its flop. The different partition functions are speculatively interpreted as counting stable objects in the derived category of $A$-modules under different stability conditions; their relationship should then be an instance of wall crossing in the space of stability conditions on this triangulated category.
\end{abstract}

$14 \mathrm{~J} 32 ; 14 \mathrm{~N} 10$

\section{Introduction}

This paper is concerned with phenomena in the enumerative geometry of local CalabiYau threefolds, mostly via a study of the example $X=\mathcal{O}_{\mathbb{P}^{1}}(-1,-1)$, a toric Calabi-Yau variety often referred to as the resolved conifold. It is known that topological string theory associates a function $Z_{X}$ of two variables $(q, t)$ to $X$, its topological string partition function, admitting the following infinite product form:

$$
Z_{X}(q, t)=M(-q)^{2} \prod_{k \geq 1}\left(1-(-q)^{k} e^{-t}\right)^{k},
$$

where the MacMahon function $M$ is itself an infinite product

$$
M(q)=\prod_{k \geq 1}\left(1-q^{k}\right)^{-k}
$$

According to Maulik et al [23], the function $Z_{X}$ admits two different Laurent series expansions, with coefficients which are enumerative invariants associated to $X$. The 
expansion in the variables $\left(q, e^{-t}\right)$ gives coefficients which are Donaldson-Thomas invariants, defined as the virtual number of singular $U(1)$-connections (more precisely rank-1 torsion free sheaves) on $X$, evaluated by enumetating certain combinatorial arrangements related to 3-dimensional partitions (compare Section 3.1). On the other hand, dividing by the MacMahon factor, we can expand in variables $\left(u, e^{-t}\right)$, with the new variable $u$ related to $q$ via $q=-e^{i u}$. The coefficients in this case are the (disconnected) Gromov-Witten invariants of $X$, counting stable maps of (not necessarily connected) curves to $X$ of arbitrary genus and degree.

In the language of string theory, the variable $u$ is the string coupling constant. The two expansions are in the parameter region (large $t$, small $u$ ), where perturbative string theory gives a good description and hence the coefficients are Gromov-Witten invariants, and (large $t$, large $|u|$ ), the region where the physical description is (conjecturally) a version of $U(1)$ gauge theory (Iqbal et al [18]), leading to rank-1 Donaldson-Thomas invariants. This leads to an obvious question: what happens in parameter regions where $t$ is small?

The parameter $t$ is geometric: it is the Kähler class, measuring the volume of the zero-section $\mathbb{P}^{1} \cong C \subset X$. Hence the limit $t \rightarrow 0$ corresponds to contracting the zero-section, resulting in the conifold singularity $Z=\left\{x_{1} x_{2}-x_{3} x_{4}=0\right\} \subset \mathbb{C}^{4}$. This singular variety is not known to have sensible enumerative invariants. I consider instead its non-commutative resolution (Van den Bergh [5]), a homologically smooth non-commutative Calabi-Yau algebra $A$, and show that this algebra gives rise to a set of enumerative invariants analogous to the Donaldson-Thomas invariants of $X$. These invariants are combinatorial, like those of $X$, this time given by counting finite subsets of a certain rectangular arrangement of two-coloured stones, or equivalently, infinite dimer configurations on the square lattice with fixed asymptotics. Using a combinatorial result due to Young [29], originally formulated as a conjecture in an earlier version of this paper, a non-perturbative change of variables leads to a partition function with a product expansion

$$
Z_{A}(q, t)=M(-q)^{2} \prod_{k \geq 1}\left(1-(-q)^{k} e^{-t}\right)^{k}\left(1-(-q)^{k} e^{t}\right)^{k},
$$

closely related to that of $Z_{X}$, which however only admits a Taylor expansion near $e^{-t}=1$ or $t=0$. Thus, in this parameter region, a new phenomenon emerges: the function $Z_{X}$ is roughly "the positive half" of the full function $Z_{A}$, which is given naturally by non-commutative geometry and dimer models.

Dimers have been in fashion lately in the high energy physics literature, already making an occurence in Okounkov-Reshetikhin-Vafa [24], and independently in work 
of Hanany and coauthors [16; 13] studying gauge/string duality for toric CalabiYau singularities. However, their direct appearance in the mathematical literature, connecting them to enumerative invariants of non-commutative Calabi-Yau geometries, seems to be new. It would be interesting to relate this idea to the other direct derivation of dimer models from toric geometry by Feng et al [12], based on mirror symmetry and special Lagrangian geometry.

Section 1 studies quiver algebras defined by a superpotential, of which the conifold algebra $A$ is an example, proving in Section 1.3 the basic fact that moduli spaces of framed cyclic modules over such algebras (the exact analogues of rank-1 torsion-free sheaves, or rather the corresponding quotient sheaves) admit a perfect obstruction theory. The obstruction theory is constructed via natural embeddings of these highly singular moduli spaces into smooth varieties; the existence of these embeddings may be of independent interest.

Section 2 is about the non-commutative conifold, reducing the computation of its enumerative invariants to combinatorics and dimer configurations in Section 2.5-Section 2.6, and discussing the product form of the partition function in Section 2.7.

Section 3 discusses possible interpretations and generalizations. The natural space parametrized by the variable $z=e^{-t}$ is introduced in Section 3.2; this is the space of Bridgeland stability conditions of a variant of the derived category of coherent sheaves on $X$. The statement that the limit $t \rightarrow 0$ should be thought of as moving to the non-commutative resolution $A$ becomes more precise on this space. A speculative interpretation of the partition functions $Z_{X}, Z_{A}$ as counts of stable objects in the derived category is also given there. This points towards an interpretation of the change from $Z_{X}$ to $Z_{A}$ as a result of performing a countably infinite number of wall crossings in the space of stability conditions.

A curious expansion of the partition function $Z_{A}$ near small $t$, involving Eisenstein-like sums, is discussed in Section 3.3. In Section 3.4 an obvious combinatorial generalization of the partition function is studied, though the enumerative interpretation of this generalization is currently unclear. Section 3.5 presents a way of extending the problem studied here to a global situation. The concluding Section 3.6 briefly discusses work of Bryan-Young [30] on some orbifold examples, where the partition function exhibits the same "doubling" phenomenon when moving from the commutative resolution to the non-commutative one. 


\section{Non-commutative Donaldson-Thomas theory}

\subsection{Quiver algebra and the superpotential}

Let $Q=\{V, E\}$ be a quiver with vertex set $V$, edge set $E$ and head and tail maps $h, t: E \rightarrow V$ prescribing the head and tail of each oriented edge (which may in general coincide). Let $\mathbb{C} Q$ be the path algebra of $Q$, generated over $\mathbb{C}$ by oriented paths, with multiplication defined by joining paths. The vector space $\mathbb{C} Q /[\mathbb{C} Q, C Q]$ has as basis the set of cycles up to cyclic permutation of the arrows. Given a superpotential, an element $W \in \mathbb{C} Q /[\mathbb{C} Q, \mathbb{C} Q]$, define a two-sided ideal $I_{W}$ of $\mathbb{C} Q$ by

$$
I_{W}=\left\langle\left\langle\partial_{e} W \mid e \in E\right\rangle\right\rangle .
$$

Here represent $W$ as an element in the path algebra consisting of a sum of cycles, well-defined up to cyclic permutation. Define formal differentiation $\partial_{e}$ of a cyclic monomial with respect to an edge $e \in E$ to be zero if $e$ does not appear in the monomial; otherwise, cyclically permute the monomial until the edge $e$ is in the first position, and delete it. For details, see eg Bocklandt [7] and Ginzburg [14]. Let $A=\mathbb{C} Q / I_{W}$ be the quotient of the path algebra $\mathbb{C} Q$ by the ideal $I_{W}$ of relations defined by the superpotential $W$.

The algebra $A$ contains a set $\left\{f_{i}: i \in V\right\}$ of orthogonal idempotents based at the vertices of $Q$, represented by paths of length 0 . Thus $A$ decomposes as $A=\oplus_{i \in V} A f_{i}$ into a set $P_{i}=A f_{i}$ of projective left $A$-modules. Note that here and everywhere below, juxtaposition denotes multiplication in the algebra $A$, or in an $A$-module, as appropriate. The algebra $A$ is generated over the commutative idempotent ring $\mathbb{C}\left[f_{i}: i \in V\right]$ by non-commuting variables $\left\{x_{e}: e \in E\right\}$ attached to the arrows of the quiver, which satisfy the relations obtained by formal differentiation of $W$ with respect to the variables $x_{e}$. As $A$ is a path algebra of a quiver with relations, the category $A$-mod of finite-dimensional left $A$-modules is equivalent to the category $\operatorname{rep}_{I_{W}}(Q)$ of finite-dimensional representations $\left(M_{i}, \varphi_{e}\right)$ of the quiver satisfying the relations $I_{W}$. I will switch between the two languages freely without comment. Objects in $\operatorname{rep}_{I_{W}}(Q)$ have a dimension vector $\mathbf{v}=\left\{v_{i}=\operatorname{dim} M_{i}\right\} \in \mathbb{Z}^{V}$.

Let $M$ be a cyclic left $A$-module, generated by an element $m \in M$; thus $M=A m$. Then $M=\oplus_{i \in V} A m_{i}$ where $m_{i}=f_{i} m$. A cyclic module generated by a vector $m \in f_{k} M$ will be called a cyclic module based at the vertex $k \in V$.

Example 1.1.1 Consider the quiver $Q=\{V, E\}$ with one vertex $V=\{*\}$, three loop edges $E=\left\{x_{1}, x_{2}, x_{3}\right\}$ with heads and tails at the given vertex $*$, and cubic superpotential

$$
W=x_{1} x_{2} x_{3}-x_{1} x_{3} x_{2}
$$


The quiver algebra

$$
\mathbb{C} Q=\mathbb{C}\left\langle x_{1}, x_{2}, x_{3}\right\rangle
$$

is a free non-commutative $\mathbb{C}$-algebra on three generators. The ideal $I_{W}$ in $\mathbb{C} Q$ is generated by the relations

$$
\begin{aligned}
& \partial_{x_{1}} W=x_{2} x_{3}-x_{3} x_{2}, \\
& \partial_{x_{2}} W=x_{3} x_{1}-x_{1} x_{3}, \\
& \partial_{x_{3}} W=x_{1} x_{2}-x_{2} x_{1} .
\end{aligned}
$$

Thus

$$
A=\mathbb{C}\left\langle x_{1}, x_{2}, x_{3}\right\rangle /\left\langle x_{i} x_{j}-x_{j} x_{i}\right\rangle \cong \mathbb{C}\left[x_{1}, x_{2}, x_{3}\right]
$$

is commutative, the coordinate ring of affine space $\mathbb{C}^{3}$. The dimension lattice of $A$ is $\mathbb{Z}$. A more substantial example, relevant to the rest of the paper, is discussed in Section 2.1 .

\subsection{The moduli space of cyclic modules}

Let $A$ be an algebra defined by a quiver $Q=\{V, E\}$ with superpotential $W$. Fix a vertex $k \in V$, as well as vector spaces $\left\{U_{i}: i \in V\right\}$ with dimension vector $\mathbf{v} \in \mathbb{Z}^{V}$. Consider the vector space

$$
S=\left(\prod_{e \in E} \operatorname{Hom}\left(U_{t(e)}, U_{h(e)}\right)\right) \times U_{k}=\left\{\left(\varphi_{e}\right)_{e \in E}, m\right\} .
$$

Let $S^{0} \subset S$ be the open subvariety defined by the condition that the vector $m \in U_{k}$ generates the $\mathbb{C} Q$-module $\left(U_{i}, \varphi_{e}\right)$. Let

$$
X=\left\{\partial_{e} W=0 \mid e \in E\right\} \subset S^{0}
$$

be the closed subscheme of $S^{0}$ cut out by the superpotential equations.

The group $G=\prod_{i \in V} \operatorname{GL}\left(U_{i}\right)$ acts on $S$ by

$$
\left(g_{i}\right) \circ\left(\left(\varphi_{e}\right), m\right)=\left(\left(g_{h(e)} \varphi_{e} g_{t(e)}^{-1}\right), g_{k} m\right),
$$

where $h, t: E \rightarrow V$ are the head and tail maps. Lift the action to $S \times \mathbb{C}$ by

$$
\left(g_{i}\right) \circ\left(\left(\varphi_{e}\right), m, z\right)=\left(\left(g_{h(e)} \varphi_{e} g_{t(e)}^{-1}\right), g_{k} m, \chi^{-1}\left(g_{i}\right) z\right),
$$

where the character $\chi$ of $G$ is defined by $\chi\left(g_{i}\right)=\prod_{i \in V} \operatorname{det}\left(g_{i}\right)$. The following lemma is now standard (King [21]). 


\section{Lemma 1.2.1}

(1) The action of $G$ on $S^{0}$ is free.

(2) For a point $\left(\left(\varphi_{e}\right), m, z\right) \in S \times \mathbb{C}$ with $z \neq 0$, the closure of the orbit

$$
\overline{G\left(\left(\varphi_{e}\right), m, z\right)} \subset S \times \mathbb{C}
$$

is disjoint from the zero-section $S \times\{0\}$ if and only if $\left(\left(\varphi_{e}\right), m\right) \in S^{0}$.

Proof For (1), if $g \in G$ fixes $\left(\left\{\varphi_{e}\right\}, m\right) \in S^{0}$, then $\operatorname{ker}\left(g-\right.$ id) $\subset \oplus_{i \in V} U_{i}$ is a subspace containing $m$ and stable under $\left\langle\varphi_{j}\right\rangle$. Thus, by definition, $g=$ id. Hence the action of $G$ on $S^{0}$ is free.

For (2), suppose that $\left(\left(\varphi_{e}\right), m\right)$ is not cyclic. Then there are decompositions $U_{i}=$ $V_{i} \oplus W_{i}$ with

$$
\varphi_{e}=\left(\begin{array}{cc}
* & * \\
0 & *
\end{array}\right) \text { and } m=\left(\begin{array}{c}
* \\
0
\end{array}\right)
$$

with respect to this decomposition. For $s \in \mathbb{C}^{*}$, let

$$
g_{i}(s)=\left(\begin{array}{cc}
1 & 0 \\
0 & \operatorname{diag}\left(s^{-1}\right)
\end{array}\right)
$$

then

$$
\left(g_{e}(s)\right) \circ\left(\left(\begin{array}{cc}
* & * \\
0 & *
\end{array}\right), m, z\right)=\left(\left(\begin{array}{cc}
* & s * \\
0 & *
\end{array}\right), m, s^{N} z\right)
$$

with $N>0$, having a limit at $s=0$ on the zero-section.

Conversely, assume that $\left(\left(\varphi_{e}\right), m\right)$ is cyclic, but the closure of the orbit $G\left(\left(\varphi_{e}\right), m, z\right)$ intersects the zero-section. Then there is a one-parameter subgroup $\lambda: \mathbb{C}^{*} \rightarrow G$ with

$$
\lim _{s \rightarrow 0} \lambda(s) \circ\left(\left(\varphi_{e}\right), m, z\right) \in S \times\{0\} .
$$

Decompose $U_{i}=\oplus_{n \in \mathbb{Z}} U_{i, n}$ under the action of $\mathbb{C}^{*}$. Since the limit at $s=0$ exists, $m \in \oplus_{n \geq 0} U_{i, n}$, and $\varphi_{e}: U_{t(e), m} \rightarrow \oplus_{n \geq 0} U_{h(e), m+n}$. Thus, since $\left(\left(\varphi_{e}\right), m\right)$ is cyclic, $U_{i}=\oplus_{n \geq 0} U_{i, n}$. Hence $\chi(\lambda(s))=s^{N}$, with $N \geq 0 . N=0$ is impossible, since then the limit would be contained in the orbit. If $N>0$, then

$$
\lambda(s) \circ\left(\left(\varphi_{e}\right), m, z\right)=\left(\left(\lambda_{h(e)}(s) \varphi_{e} \lambda_{t(e)}^{-1}(s)\right), \lambda(s) m, s^{-N_{z}}\right)
$$

does not converge to a point on the zero-section as $s \rightarrow 0$, a contradiction. 


\section{Proposition 1.2.2}

(1) There exists a smooth and quasi-projective geometric quotient $N$ of $S^{0}$ by $G$, containing a closed subscheme $\mathcal{M}_{k, \mathbf{v}} \subset N$ which is a quotient of $X$ by $G$.

(2) The space $\mathcal{M}_{k, \mathbf{v}}$ carries a tautological family $\left(M_{k, \mathbf{v}}, m_{k, \mathbf{v}}\right)$ of framed cyclic $A$-modules, generated at the vertex $k$.

(3) The space $\mathcal{M}_{k, \mathbf{v}}$ a fine moduli space; the triple $\left(\mathcal{M}_{k, \mathbf{v}}, M_{k, \mathbf{v}}, m_{k, \mathbf{v}}\right)$ represents the functor of flat families of framed $A$-modules (locally free sheaves with $A$-structure and $A$-generator) over schemes, generated by a section based at the vertex $k$.

Proof To prove (1), following [21], regard the affine space $S$ as a quasi-projective variety, and consider the linearization (1) of the action of $G$ on the trivial line bundle $S \times \mathbb{C}$. Lemma 1.2.1 (2) implies that the semistable locus $S^{\text {ss }}$ is exactly the subset $S^{0}$. This also agrees with the stable locus, since all stabilizers of points in $S_{0}$ are trivial by Lemma 1.2.1 (1). Thus, by Geometric Invariant Theory, a quasi-projective geometric quotient $N=S / /{ }_{\chi} G=S^{0} / G$ exists, and it is smooth because stabilizers on $S^{0}$ are trivial. Since $X$ is $G$-invariant in $S^{0}$, its quotient by $G$ exists as a closed subscheme $\mathcal{M}_{k, \mathbf{v}} \subset N$.

For (2), it is enough to observe that, tautologically, $S^{0}$ carries a tautological family of $\mathbb{C} Q$-modules, generated by a section. Over $X \subset S^{0}$, the relations are also satisfied, and hence the family becomes that of $A$-modules. Being $G$-equivariant, the family and its generator descend to the space $\mathcal{M}_{k, \mathbf{v}}$.

Finally, to see (3), let $Z$ be a scheme with a flat family $M_{Z}$ of $A$-modules, locally free sheaves with $A$-structure, generated by a section $m \in H^{0}\left(Z, M_{Z}\right)$. Taking trivializations of $M_{Z}$ on an open cover $\left\{Z_{j}\right\}$ of $Z$ gives tautological maps $Z_{j} \rightarrow X$, and composing with the projection $X \rightarrow \mathcal{M}_{k, \mathbf{v}}$, these maps glue to a map $Z \rightarrow$ $\mathcal{M}_{k, \mathrm{v}}$ under which the family on $Z$ is a pullback by construction. This shows that $\left(\mathcal{M}_{k, \mathbf{v}}, M_{k, \mathbf{v}}, m_{k, \mathbf{v}}\right)$ is indeed a universal family.

At a point $[M, m] \in \mathcal{M}_{k, \mathbf{v}}$, let $\bar{m}: A \rightarrow M$ be the canonical $A$-module surjection given by $a \mapsto a m$. Let $I=\operatorname{ker} \bar{m}$. Since $m \in f_{k} M$, where $f_{k}$ is the idempotent based at the $k$-th vertex, the ideal $I$ decomposes as a direct sum $I=I_{k} \oplus \bigoplus_{j \neq k} P_{j}$, into the left $A$-ideal $I_{k}=I f_{k}$ and the remaining projective modules $P_{j}=A f_{j}$ for $j \neq k$.

Corollary 1.2.3 The Zariski tangent space to $\mathcal{M}_{k, \mathbf{v}}$ at its point $[M, m]$ can be identified with the $\mathbb{C}$-vector space $\operatorname{Hom}_{A}\left(I_{k}, M\right)$. 
Proof Each deformation $\left(M_{\eta}, m_{\eta}\right)$ of $(M, m)$ in $\mathcal{M}_{k, \mathbf{v}}$ is still based at the vertex $k$. Hence the projectives $P_{j}$ for $j \neq k$ must remain in the kernel of $\bar{m}_{\eta}$. With this modification, the statement is a standard corollary of Proposition 1.2.2 (2); see eg Artin-Zhang [1, Proposition E2.4(ii)].

\subsection{Perfect obstruction theory on the moduli space}

Recall from Behrend-Fantechi [3] that given a scheme $Z$, a perfect obstruction theory $(E, \psi)$ for $Z$ is morphism $\psi: E \rightarrow L_{Z}$ in the derived category $\mathcal{D}(Z)$ of quasicoherent $\mathcal{O}_{Z}$-modules, where $L_{Z}$ is the cotangent complex of $Z, E \in \mathcal{D}(Z)$ is a perfect complex of amplitude $[-1,0]$, and $\psi$ induces an isomorphism on $H^{0}$ and a surjection on $H^{-1}$. A symmetric perfect obstruction theory (Behrend-Fantechi [4]) is a triple $(E, \psi, \theta)$ including also a non-degenerate symmetric bilinear form $\theta: E \rightarrow E^{\vee}[1]$.

Theorem 1.3.1 The moduli space $\mathcal{M}_{k, \mathbf{v}}$ carries a symmetric perfect obstruction theory.

Proof Recall the superpotential $W \in \mathbb{C} Q /[\mathbb{C} Q, \mathbb{C} Q]$ used to define the algebra $A$. Consider the regular function $w=\operatorname{Tr}(W)$ on the smooth variety $S^{0}$, obtained by taking the sum of the traces of the cycles making up $W$. As spelled out for example in Segal [25, Proposition 3.8],

$$
X=Z(d w) \subset S_{0}
$$

is exactly the scheme-theoretic vanishing locus of the one-form $d w \in \Omega_{S^{0}}^{1}$. Note that [25] deals with the case where there is no cyclic generator, but the proof carries over verbatim: the equations defining $X \subset S_{0}$ are the relations between various linear maps prescribed by the superpotential $W$; they do not involve the generator. The cyclic generator is only used to define the open subset $S_{0} \subset S$.

The function $w \in H^{0}\left(\mathcal{O}_{S^{0}}\right)$ is invariant under the $G$-action above, and hence descends to a regular function $\bar{w} \in H^{0}\left(\mathcal{O}_{N}\right)$ on the smooth quotient $N$. By naturality, $\mathcal{M}_{k, \mathbf{v}}=$ $Z(d \bar{w})$ is the vanishing locus on $N$ of the exact one-form $d \bar{w}$. By Behrend [2, Remark 3.12], this defines a symmetric perfect obstruction theory on $\mathcal{M}_{k, \mathbf{v}}$.

Remark 1.3.2 Recall that an associative $\mathbb{C}$-algebra $A$ is $3-C a l a b i-Y a u$, if for all $M, N \in A$-Mod, the category of finitely generated $A$-modules, with at least one of $M, N$ finite-dimensional, there exist perfect bifunctorial pairings

$$
\operatorname{Ext}_{A}^{i}(M, N) \times \operatorname{Ext}_{A}^{3-i}(N, M) \rightarrow \mathbb{C}
$$

between finite-dimensional $\mathbb{C}$-vector spaces. For certain choices of superpotentials, it is known that the algebra $A$ considered above is 3-Calabi-Yau (see Bocklandt [7] and 
Ginzburg [14]). The Calabi-Yau duality between Ext ${ }^{1}$ and Ext ${ }^{2}$ is morally responsible for the existence of the symmetric perfect obstruction theory on the module space of $A$-modules, though as the proof shows, it is not a necessary requirement for cyclic modules.

For the case of sheaves on a Calabi-Yau threefold, the construction of the symmetric obstruction theory is due to Thomas. The proof in Thomas [26] uses the equations of the moduli space inside a Grassmannian; this inspired the proof of Theorem 1.3.1 above. The proof in Thomas [27] uses duality explicitly.

\subsection{Cyclic modules and finite-codimension ideals}

The purpose of this section is to show that, at least set-theoretically and under the Calabi-Yau assumption, parametrizing cyclic finite-dimensional $A$-modules up to isomorphism, respecting the cyclic generator, is equivalent to parametrizing finitecodimension ideals of $A$ up to $A$-module isomorphism. This is analogous to the relationship between the moduli space of ideal sheaves and the Hilbert scheme on a projective variety. I state a precise variant of this claim, taking into account that the generator is based at a particular vertex. As before, for a vertex $j \in V$, let $f_{j} \in A$ be the idempotent based at $j$, and $P_{j}=A f_{j}$ the corresponding projective $A$-module.

Proposition 1.4.1 Assume that the algebra $A$ is 3-Calabi-Yau. Then there is a bijection between the set of finitely generated left $A$-modules, embeddable into $P_{k}$ with finite codimension, up to $A$-module isomorphism, and the pairs $(M, m)$ of finite-dimensional cyclic $A$-modules $M$ with generator $m \in f_{k} M$, up to $A$-module isomorphism respecting generators.

Proof As before, the cyclic module $(M, m)$ defines a surjection $\bar{m}: A \rightarrow M$ sending $1 \in A$ to $m \in M$. As $m \in f_{k} M$, the kernel $I=\operatorname{ker} \bar{m}$ decomposes as $I=$ $I_{k} \oplus \bigoplus_{j \neq k} P_{j}$, with the left $A$-ideal $I_{k}=I f_{k}$ embedded in $P_{k}$ with finite codimension. Isomorphic pairs give rise to isomorphic $A$-modules. Conversely, suppose that $N \in A$-Mod is embeddable into $P_{k}$ with finite-dimensional cokernel. Take such an embedding $i: N \hookrightarrow P_{k}$, and consider the exact sequence of left $A$-modules

$$
0 \rightarrow N \stackrel{i}{\rightarrow} P_{k} \rightarrow M \rightarrow 0
$$

Part of the corresponding long exact sequence of abelian groups reads

$$
\operatorname{Hom}_{A}\left(M, P_{k}\right) \rightarrow \operatorname{Hom}_{A}\left(P_{k}, P_{k}\right) \rightarrow \operatorname{Hom}_{A}\left(N, P_{k}\right) \rightarrow \operatorname{Ext}_{A}^{1}\left(M, P_{k}\right) .
$$

However, $M$ is finite dimensional, so by the Calabi-Yau duality, for $i<3$

$$
\operatorname{Ext}_{A}^{i}\left(M, P_{k}\right) \cong \operatorname{Ext}_{A}^{3-i}\left(P_{k}, M\right)^{*}=0,
$$


since $P_{k}$ is a projective $A$-module. Hence

$$
\operatorname{Hom}_{A}\left(N, P_{k}\right) \cong \operatorname{Hom}_{A}\left(P_{k}, P_{k}\right) \cong f_{k} P_{k},
$$

where the last isomorphism is given by $g \mapsto g\left(f_{k}\right) \in f_{k} P_{k}$. Thus the only other $A$-maps from $N$ to $P_{k}$ are multiples of $i$ by some $a \in f_{k} P_{k}$. These other embeddings have cokernel $P_{k} / N a$ which has $P_{k} / P_{k} a$ as quotient, the latter being infinite-dimensional unless $a \in f_{k} P_{k}$ is a constant multiple of the idempotent $f_{k}$. Hence the only embeddings of $N$ into $P_{k}$ with finite-dimensional quotient are constant multiples of $i$, and hence $i$, and thus the surjection $A \rightarrow M$ defining a pair $(M, m)$, are uniquely determined by $N$ up to $A$-isomorphism.

As usual, one can presumably promote the statement to an isomorphism of moduli spaces, once the relevant moduli problem is formulated for ideals. Since this is not relevant for the rest of the paper, I will not pursue this direction.

\subsection{Numerical invariants}

Given a scheme $Z$ which admits a symmetric perfect obstruction theory, Behrend [2] proves that there is a canonical constructible $\mathbb{Z}$-valued function $v_{Z}$ on $Z$. This allows one to define the virtual number of points of $Z$ to be the integer

$$
\#^{\mathrm{vir}}(Z)=\sum_{n \in \mathbb{Z}} n \chi\left(v_{Z}^{-1}(n)\right),
$$

where $\chi$ denotes the topological Euler characteristic. For the case of the non-commutative algebra $A$, we therefore get integers

$$
D_{k, \mathrm{v}}=\#^{\mathrm{vir}}\left(\mathcal{M}_{i, \mathrm{v}}\right),
$$

depending on a choice of base vertex $k \in V$ and dimension vector $\mathbf{v} \in \mathbb{Z}^{V}$. These can be encoded in the partition function

$$
Z_{A, k}(\mathbf{q})=\sum_{\mathbf{v} \in \mathbb{Z}^{V}} D_{k, \mathbf{v}} \mathbf{q}^{\mathbf{v}},
$$

using a set of auxiliary variables $\mathbf{q}=\left\{q_{i}: i \in V\right\}$.

\subsection{The commutative Hilbert scheme}

Recall the example discussed in Example 1.1.1, with the quiver $Q$ having one vertex $V=\{1\}$ and three loop edges, leading to $A \cong \mathbb{C}\left[x_{1}, x_{2}, x_{3}\right]$ commutative with dimension lattice $\mathbb{Z}$. For $n \geq 1$, the moduli space $\mathcal{M}_{1, n}$ is well known to be $\operatorname{Hilb}^{[n]}\left(\mathbb{C}^{3}\right)$, the Hilbert scheme of $n$ points on $\mathbb{C}^{3}$. This space carries a symmetric obstruction 
theory via its manifestation as the moduli of rank-1 sheaves with trivial determinant (see Thomas [26; 27] and Behrend-Fantechi [4]) on $\mathbb{C}^{3}$. Theorem 1.3.1 above gives a new, in some sense more elementary, construction of this obstruction theory.

The series $Z_{A, 1}(q)$ can be computed by torus localization (Okounkov et al [24], Maulik et al [23] and Behrend-Fantechi [4]), the torus-fixed points being parametrized by 3-dimensional partitions $\alpha \subset \mathbb{N}^{3}$. This gives

$$
Z_{A, 1}(q)=\sum_{\alpha}(-q)^{\mathrm{wt}(\alpha)},
$$

where $\operatorname{wt}(\alpha)$ is the total weight of the partition $\alpha$. By a classical result of MacMahon, this function is given by

$$
Z_{A, 1}(q)=M(-q)=\prod_{n \geq 1}\left(1-(-q)^{n}\right)^{-n}
$$

Recall also [24, Section 5.4] that finite 3-dimensional partitions are in one-to-one correspondence with dimer configurations in the hexagonal lattice (honeycomb dimers), with a certain boundary condition.

In the rest of the paper, I study the partition function $Z_{A}(\mathbf{q})$ for a more complicated example, obtaining analogous results.

\section{The non-commutative conifold}

\subsection{The algebra}

Consider the quiver $Q=\{V, E\}$ of Figure 1, with two vertices $V=\{0,1\}$, four oriented edges $E=\left\{a_{1}, a_{2}: 0 \rightarrow 1, b_{1}, b_{2}: 1 \rightarrow 0\right\}$, and relations coming from the quartic superpotential $W=a_{1} b_{1} a_{2} b_{2}-a_{1} b_{2} a_{2} b_{1}$ (the Klebanov-Witten superpotential [22]). Thus the algebra $A$ contains the idempotent ring $\mathbb{C}\left[f_{0}, f_{1}\right]$, and can be given by generators and relations as

$$
A=\mathbb{C}\left[f_{0}, f_{1}\right]\left\langle a_{1}, a_{2}, b_{1}, b_{2}\right\rangle /\left\langle b_{1} a_{i} b_{2}-b_{2} a_{i} b_{1}, a_{1} b_{i} a_{2}-a_{2} b_{i} a_{1}: i=1,2\right\rangle .
$$

The dimension lattice is $\mathbb{Z}^{V}=\mathbb{Z}^{2}$. The center $R=Z(A)$ is spanned by $x_{1}=$ $a_{1} b_{1}+b_{1} a_{1}, x_{2}=a_{2} b_{2}+b_{2} a_{2}, x_{3}=a_{1} b_{2}+b_{2} a_{1}, x_{4}=a_{2} b_{1}+b_{1} a_{2}$, so

$$
R=\mathbb{C}\left[x_{1}, x_{2}, x_{3}, x_{4}\right] /\left(x_{1} x_{2}-x_{3} x_{4}\right),
$$

the ring of functions on the threefold ordinary double point or conifold singularity $Z=\operatorname{Spec}(R)$. Indeed, $A$ is a 3-Calabi-Yau algebra, and a crepant non-commutative resolution (Van den Bergh [5]) of the singular variety $Z$. 


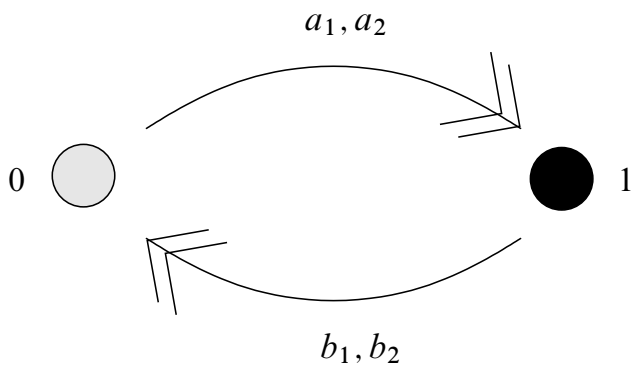

Figure 1: The conifold quiver

\subsection{Torus actions}

Let $T_{F}=\left(\mathbb{C}^{*}\right)^{E}$ be the "flavour" torus, acting by diagonally rescaling the edge variables. The relations are automatically preserved, and thus $T_{F}$ acts as a group of automorphisms of the algebra $A . T_{F}$ has two distinguished subtori. As a subgroup of $\operatorname{Aut}(A)$, it contains the "gauge" torus $\left(\mathbb{C}^{*}\right)^{V} / \mathbb{C}^{*}$, acting on $A$ by inner automorphisms as $r \mapsto\left(\sum \mu_{i}^{-1} f_{i}\right) r\left(\sum \mu_{i} f_{i}\right)$; note diagonal elements act trivially. On the other hand, $T_{F}$ also acts on the whole quiver algebra $\mathbb{C} Q$, and hence it has a subtorus $T_{F, W} \subset T_{F}$ which stabilizes the superpotential $W$.

Recall the space $X=\left\{\left(\left(\varphi_{e}\right)_{e \in E}, m\right)\right\}$ of cyclic representations of the quiver $Q$ on a collection of vector spaces $\left(U_{i}\right)_{i \in V}$, generated by a vector $m \in U_{k}$. The flavour torus $T_{F}=\left(\mathbb{C}^{*}\right)^{E}=\left\{\left(\lambda_{e}\right)_{e \in E}\right\}$ acts on $X$ by

$$
\left(\lambda_{e}\right) \circ\left(\left(\varphi_{e}\right), m\right)=\left(\left(\lambda_{e} \varphi_{e}\right), m\right) .
$$

The torus $T_{G}=\left(\mathbb{C}^{*}\right)^{V}=\left\{\left(\mu_{i}\right)_{i \in V}\right\}$ also acts on $X$, as a subgroup of $G=\prod_{i \in V} \operatorname{GL}\left(U_{i}\right)$, by

$$
\left(\mu_{i}\right) \circ\left(\left(\varphi_{e}\right), m\right)=\left(\left(\mu_{h(e)} \mu_{t(e)}^{-1} \varphi_{e}\right), \mu_{k} m\right) .
$$

The intersection of the image of $T_{F}$ and the image of $G$ in $\operatorname{Aut}(X)$ is generated by the subtorus $T_{k}=\left(\mathbb{C}^{*}\right)^{V \backslash\{k\}}$ of $T_{F}$, acting at the other vertex $j \in V \backslash\{k\}$. Hence the moduli space $\mathcal{M}_{k, \mathbf{v}}$ of framed cyclic representations of $A$ is acted on by the quotient torus $T=T_{F} / T_{k}$ of rank 3 . The latter has a subtorus $T_{W}=T_{F, W} / T_{k} \cap T_{F, W}$ which stabilizes the regular function $\bar{w} \in H^{0}\left(\mathcal{O}_{N}\right)$ used in the construction of the perfect obstruction theory on $\mathcal{M}_{k, \mathbf{v}} \subset N$.

Explicitly, $T$ can be described as the quotient of the four-dimensional torus $\left(\mathbb{C}^{*}\right)^{4}$ acting on the edge variables $a_{i}, b_{j}$ by the subtorus $\mathbb{C}^{*}$ acting by weights $(-1,-1,1,1)$. $T_{W} \subset T$ is defined by the condition that the product of the elements is 1 . 


\subsection{The resolution as moduli space}

Let us construct one well known moduli space of cyclic $A$-modules, that based at the vertex 0 , corresponding to the dimension vector $(1,1) \in \mathbb{Z}^{2}$. Choosing bases leads to $U_{i} \cong \mathbb{C}$ and then the arrows $a_{1}, a_{2}, b_{1}, b_{2} \in \mathbb{C}$ as well as the generator $m \in \mathbb{C}^{*}$ are scalars; cyclicity requires that not both $a_{1}, a_{2}$ should be zero. Thus

$$
\begin{aligned}
\mathcal{M}_{0,(1,1)} & \cong\left(\mathbb{C}_{a_{i}, b_{j}}^{4} \times \mathbb{C}_{m}^{*} \backslash\left\{a_{i}=0\right\}\right) / \mathbb{C}^{*}(-1,-1,1,1 ; 1) \times \mathbb{C}^{*}(1,1,-1,-1 ; 0) \\
& \cong\left(\mathbb{C}_{a_{i}, b_{j}}^{4} \backslash\left\{a_{i}=0\right\}\right) / \mathbb{C}^{*}(-1,-1,1,1)
\end{aligned}
$$

which is well known to be isomorphic to the crepant resolution $X$ of the singular conifold $Z=\operatorname{Spec}(R)$.

\subsection{Pyramid partitions}

Consider the infinite combinatorial arrangement (Kenyon [20]) on Figure 2, with two types of layers of stones. For $i \geq 0$, there are $(i+1)^{2}$ stones labelled 0 and coloured grey on layer $2 i$. On layer $2 i+1$, there are $(i+1)(i+2)$ stones labelled 1 and coloured black.

A finite subset $\pi$ of the combinatorial arrangement is a pyramid partition, if for every stone in $\pi$, the (usually two) stones immediately above it, of different colour, are also contained in $\pi$. For a pyramid partition $\pi$, define $\mathrm{wt}_{i}(\pi)$ to be the number of stones labelled $i$ in $\pi$ for $i=0,1$. Denote by $\mathcal{P}$ the set of all pyramid partitons.

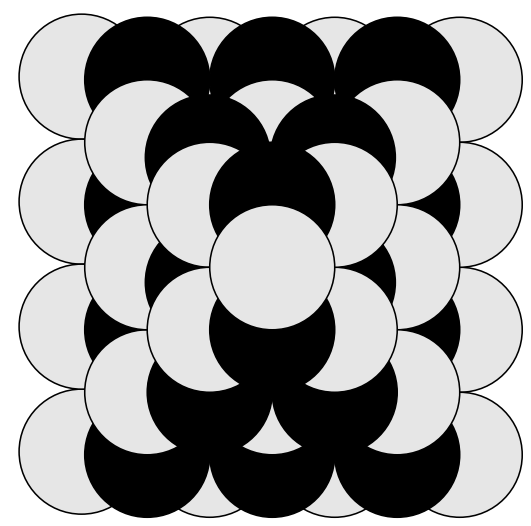

Figure 2: The pyramid arrangement

Remark 2.4.1 The subset of the combinatorial arrangement of Figure 2 consisting of grey stones forms a finitely generated semigroup $\sigma_{\mathbb{Z}}$, the intersection of $\mathbb{Z}^{3}$ with 
a polyhedral cone $\sigma$. The semigroup ring $\mathbb{C}\left[\sigma_{\mathbb{Z}}\right]$ is nothing else but the ring $R$, the center of $A$; this is the toric description of the singular conifold.

The effect of the non-commutative resolution is to introduce the odd layers of stones in the picture. The full pyramid defines the projective left $A$-module $P_{0}=A f_{0}$, where $f_{0} \in A$ is the idempotent corresponding to the chosen vertex $0 \in V$. Namely, $P_{0}$ has, as a $\mathbb{C}$-vector space, a basis labelled by all the stones in the pyramid, and the multiplication by the basic monomials $a_{1}, a_{2}, b_{1}, b_{2}$ is also encoded (see Figure 3 below). This is analogous to the way the non-negative octant in the lattice $\mathbb{Z}^{3}$ gives a basis for the commutative algebra $\mathbb{C}\left[x_{1}, x_{2}, x_{3}\right]$, with the monomials $x_{1}, x_{2}, x_{3}$ multiplying along the edges (compare Example 1.1.1 as well as Section 1.6).

\subsection{Torus-fixed points and pyramid partitions}

The two vertices of the conifold quiver $Q=\{V, E\}$ are symmetric under an outer automorphism of the algebra; thus I concentrate on cyclic $A$-modules based on the vertex $0 \in V$, and drop the index 0 from the notation. (See the end of Section 3.2 for more discussion of this choice.)

Consider the action of the rank-two torus $T_{W}$ of Section 2.2 on some moduli space $\mathcal{M}_{\mathbf{v}}$ of framed cyclic $A$-modules.

\section{Proposition 2.5.1}

(1) There are finitely many $T_{W}$-fixed points on the moduli space $\mathcal{M}_{\mathbf{v}}$. There is a one-to-one correspondence between fixed points and pyramid partitions $\pi \in \mathcal{P}$ of weight $\left(\mathrm{wt}_{1}(\pi), \mathrm{wt}_{2}(\pi)\right)=\mathbf{v} \in \mathbb{Z}^{2}$.

(2) At each fixed point $P \in \mathcal{M}_{\mathbf{v}}$, the Zariski tangent space to $\mathcal{M}_{\mathbf{v}}$ at $P$ has no $T_{W}$-invariant subspace.

Proof Suppose that $[(M, m)] \in \mathcal{M}_{\mathbf{v}}$ is an isomorphism class of framed cyclic $A-$ modules fixed by the torus $T_{W}$, represented by a framed cyclic $A$-module $(M, m)$ which can be assumed to be non-zero. Let $I \triangleleft A$ be the annihilator of the cyclic generator $m \in M$, a left ideal in $A$. As in Section 1.4, since the generating vector $m \in M$ is based at the vertex 0 , we can write $I=I_{0} \oplus P_{1}$, where $P_{1}=A f_{1}$ consists of all paths starting at the vertex 1 . First I claim that $I_{0}$ is a monomial ideal: its generators over $\mathbb{C}\left[f_{0}\right]$ are monomials in the edge variables $a_{i}, b_{j}$.

To show this, recall that explicitly, as acting on ideals in $A$, the rank-two torus $T_{W}$ manifests itself as a quotient

$$
T_{W}=T_{F, W} / \mathbb{C}^{*}
$$


of the rank-three torus

$$
T_{F, W}=\left\{\left(\lambda_{1}, \lambda_{2}, \mu_{1}, \mu_{2}\right): \lambda_{1} \lambda_{2} \mu_{1} \mu_{2}=1\right\}
$$

acting on the variables $\left(a_{1}, a_{2}, b_{1}, b_{2}\right)$, by the "gauge" subtorus

$$
\mathbb{C}^{*}=\left\{\left(\lambda, \lambda, \lambda^{-1}, \lambda^{-1}\right)\right\} \subset T_{F, W} .
$$

Generators of $I_{0}$ split up into sums of paths with the same starting point and endpoint, and on elements of $\mathbb{C} Q$ represented by such paths, this subtorus has a constant diagonal action. Hence the covering torus $T_{F, W}$ also acts on ideals, and the $T_{W}$-fixed points in $\mathcal{M}_{\mathbf{v}}$ are in one-to-one correspondence with $T_{F, W}$-fixed left $A$-ideals $I=I_{0} \oplus P_{1}$.

If the left $A$-ideal $I=I_{0} \oplus P_{1}$ is fixed by $T_{F, W}$, then it must be generated by $T_{F, W}$-eigenvectors. Let $r\left(a_{1}, a_{2}, b_{1}, b_{2}\right)$ be a (non-commutative) polynomial in the generators of $A$, which is a $T_{F, W}$-eigenvector in $I_{0}$. Note that, using the relations coming from the superpotential $W$,

$$
b_{2} a_{2} b_{1} a_{1}=b_{2} a_{1} b_{1} a_{2}=b_{1} a_{2} b_{2} a_{1}=b_{1} a_{1} b_{2} a_{2} \in A .
$$

This element $c=b_{i} a_{j} b_{3-i} a_{3-j} \in P_{0}$ commutes with all elements of $P_{0}$, and generates the weight- 0 eigenspace of $T_{F, W}$ acting on $P_{0}$. Moving the highest possible power of $c$ to the right in each monomial making up $r$, it follows that

$$
r\left(a_{i}, b_{j}\right)=q\left(a_{1}, a_{2}, b_{1}, b_{2}\right) \cdot p(c) \in A,
$$

where $p$ is a polynomial with nonzero constant term, and $q$ is a monomial in the generators $a_{i}, b_{j}$. Let $J=I_{0} \cap Z(A) f_{0}$, where $Z(A)$ is the center of $A$, and $f_{0}$ is the idempotent at the vertex 0 . Then $J$ is an ideal in $Z(A) f_{0} \cong Z(A)$, the coordinate ring of the conifold singularity. Since $I$ is fixed by $T_{W}$, the ideal $J$ has to be fixed as well, and hence the zero-set of $J$ must be supported at the singularity $0 \in \operatorname{Spec} Z(A)$. Thus this zero-set is disjoint from the zero-set of $p(c) \in Z(A) f_{0}$. By the Nullstellensatz, this implies that $\langle p, J\rangle=Z(A) f_{0}$, and hence $q \in I_{0}$. So indeed, $I_{0}$ is generated by monomials.

Consider the generator $m \in M$, and the set of vectors

$$
\mathcal{S}=\left\{m, a_{1} m, a_{2} m, b_{1} a_{1} m, b_{2} a_{1} m, b_{1} a_{2} m, b_{2} a_{2} m, \ldots\right\} \subset M .
$$

Since $m$ is a generator, the nonzero vectors in $\mathcal{S}$ form a spanning set for $M$. Certain sets of vectors in $\mathcal{S}$ will be equal in $M$ as a consequence of the relations among the $a_{i}, b_{j}$; delete all but one vector from each equivalence class. Since all remaining relations, contained in the ideal $I$, are monomial, the remaining set of nonzero vectors is linearly independent and hence forms a basis of $M$, finite since $M$ is finite dimensional. These nonzero vectors form a finite pyramid partition $\pi \in \mathcal{P}$. 
Conversely, given a pyramid partition $\pi \in \mathcal{P}$, let $M_{i}$ for $i=0,1$ be the $\mathbb{C}$-vector space spanned by basis vectors $e_{s}$ for each stone $s \in \pi$ of label $i$. The arrows $a_{i}, b_{j}$ act by mapping between basis vectors as in Figure 3. It is immediately seen that the relations in $A$ are satisfied. Hence this rule defines an $A$-module structure on $M=M_{0} \oplus M_{1}$, generated by $m=e_{0} \in M_{0}$ corresponding to the topmost stone of label 0 . The action of the torus $T_{W}$ can be compensated for by a change of basis, and hence all these modules are in the fixed locus $\left(\mathcal{M}_{\mathrm{v}}\right)^{T_{W}}$. This gives the inverse correspondence, concluding the proof of (1).

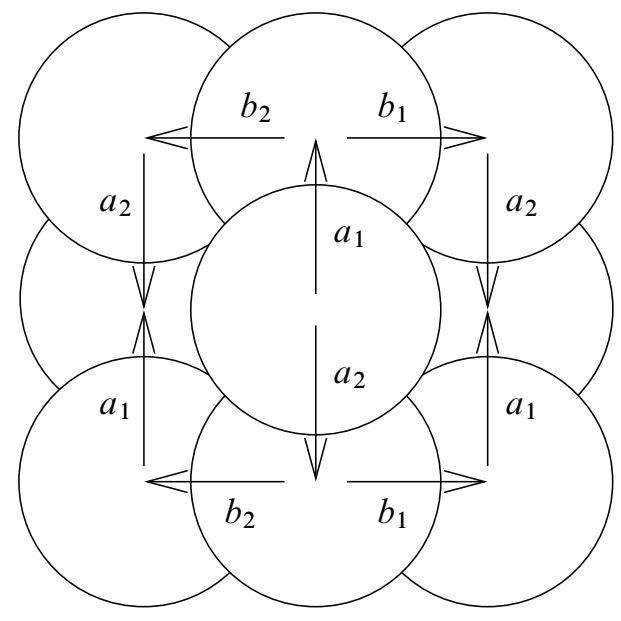

Figure 3: A pyramid partition defines a module

To prove (2), by Corollary 1.2.3, it remains to study the $T_{F, W}$-action on the space $\operatorname{Hom}_{A}\left(I_{0}, M\right)$ for a monomial ideal $I=I_{0} \oplus P_{1}$ with quotient $M=A / I$. I follow the argument of [4, Lemma 4.1]. It is enough to show that under the induced action of the "flavour" torus $T_{F}=\left(\mathbb{C}^{*}\right)^{E}$, no weight is a multiple of $(1,1,1,1)$.

Suppose therefore that $\varphi: I_{0} \rightarrow M$ is an eigenvector with weight $w(1,1,1,1)$. Suppose first that $w \geq 0$. As the $(1,1,1,1)$ eigenspace of $P_{0}$ is spanned by the element $c=b_{i} a_{j} b_{3-i} a_{3-j}$ already used above, for any element $a \in I_{0}$, necessarily

$$
\varphi(a) \equiv c^{w} a \equiv 0 \bmod I,
$$

and so $\varphi=0$.

To treat the case $w<0$, let $\alpha$ be the smallest positive integer such that $\left(b_{1} a_{1}\right)^{\alpha} \in I_{0}$, and let $\beta$ be the smallest positive integer such that $\left(b_{2} a_{2}\right)^{\beta}\left(b_{1} a_{1}\right)^{\alpha-1} \in I_{0}$. Since $\varphi$ is an $T_{W}$-eigenvector with weight $w(1,1,1,1)$,

$$
\varphi\left(\left(b_{2} a_{2}\right)^{\beta}\left(b_{1} a_{1}\right)^{\alpha-1}\right) \equiv\left(b_{2} a_{2}\right)^{\beta+w}\left(b_{1} a_{1}\right)^{\alpha-1+w} \bmod I .
$$


Now compute, using the fact that $b_{1} a_{1}$ commutes with $b_{2} a_{2}$ :

$$
\begin{aligned}
\varphi\left(\left(b_{2} a_{2}\right)^{\beta}\left(b_{1} a_{1}\right)^{\alpha}\right)=b_{1} a_{1} \varphi\left(\left(b_{2} a_{2}\right)^{\beta}\left(b_{1} a_{1}\right)^{\alpha-1}\right) & \equiv b_{1} a_{1}\left(b_{2} a_{2}\right)^{\beta+w}\left(b_{1} a_{1}\right)^{\alpha+w-1} \\
& \equiv\left(b_{2} a_{2}\right)^{\beta+w}\left(b_{1} a_{1}\right)^{\alpha+w} \bmod I .
\end{aligned}
$$

On the other hand,

$$
\varphi\left(\left(b_{1} a_{1}\right)^{\alpha}\right) \equiv 0 \bmod I,
$$

since there is no monomial in $P_{0}$ with negative $T_{W}$-weights. Hence

$$
\varphi\left(\left(b_{2} a_{2}\right)^{\beta}\left(b_{1} a_{1}\right)^{\alpha}\right)=\left(b_{2} a_{2}\right)^{\beta} \varphi\left(\left(b_{1} a_{1}\right)^{\alpha}\right) \equiv 0 \bmod I .
$$

Comparing the two expressions,

$$
\left(b_{2} a_{2}\right)^{\beta+w}\left(b_{1} a_{1}\right)^{\alpha+w} \in I .
$$

Since $w<0$, this contradicts the definition of $\beta$.

I will later need the following additional information on fixed points. Recall from Section 1.2 the construction of the moduli space $\mathcal{M}_{\mathbf{v}}$ of framed cyclic modules as the free quotient of an locally closed subset $X \subset S^{0} \subset S$ of a vector space $S$ of linear maps by a group $G$.

Lemma 2.5.2 Let $\pi \in \mathcal{P}$ be a pyramid partition, and $M_{\pi}$ the framed cyclic module defined by $\pi$. Then at the point $\left[M_{\pi}\right] \in X$, the parities of the tangent spaces $\operatorname{dim} T_{\left[M_{\pi}\right]} X$ and $\operatorname{dim} T_{\left[M_{\pi}\right]} S$ coincide.

Proof In the prescription given in the proof of Proposition 2.5.1, the $A$-module $M_{\pi}$ has a vector space basis indexed by the stones of the partition $\pi$. The affine space $S$ inherits natural coordinates given by the corresponding matrix entries. In this coordinate system, $\left[M_{\pi}\right] \in S$ has coordinates 0 and 1 as dictated by the partition $\pi$; in Figure 3, the arrows drawn correspond to coordinates equal to 1 , the rest being 0 .

The embedding $X \subset S$ is locally defined by the superpotential equations $d \operatorname{Tr}(W)$. Writing these in the matrix entries, we get cubic equations for every pair of basis vectors indexed by stones $i, l \in \pi$ of opposite colours, and appropriate composable arrows $a, b, c$ of the conifold quiver, with $a$ and $c$ different, of the form

$$
[i l ; a b c]: \sum_{j, k} a_{i j} b_{j k} c_{k l}=\sum_{j, k} c_{i j} b_{j k} a_{k l} .
$$

Here $j, k \in \pi$ run over all stones of appropriate colour. To get the embedding of tangent spaces, these equations have to be linearized near the point $\left[M_{\pi}\right] \in X$ with all coordinates either 0 or 1 . By changing variables to new variables $a_{i j}^{\prime}=a_{i j}-1$ 
whenever $a_{i j}=1$, the linearized form of (2) is non-zero only if at least two of the consecutive arrows $a_{i j}, b_{j k}, c_{k l}$, or $c_{i j}, b_{j k}, a_{k l}$ are non-zero. If for some $i$ and $l$, there are three nonzero arrows $a_{i j}, b_{j k}, c_{k l}$, then (by the relations) there is another set $c_{i m}, b_{m n}, a_{n l}$ of nonzero arrows, and the linearized form is

$$
[i l ; a b c]_{\operatorname{lin}}: \quad a_{i j}^{\prime}+b_{j k}^{\prime}+c_{k l}^{\prime}+\cdots=c_{i m}^{\prime}+b_{m n}^{\prime}+a_{n l}^{\prime}+\cdots ;
$$

If only two of the arrows, say $a_{i j}$ and $b_{j k}$ are nonzero, the linearized form is

$$
[i l ; a b c]_{\operatorname{lin}}: c_{k l}+\cdots=0 .
$$

In both cases, $\cdots$ represents further possible terms of the same shape.

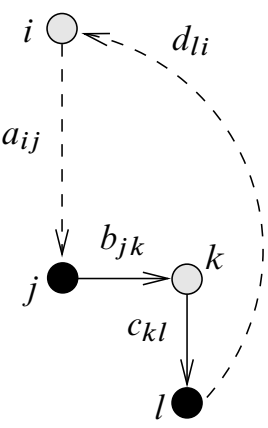

$$
\begin{aligned}
& {[i l ; a b c]_{\operatorname{lin}}:} \\
& a_{i j}+\cdots=0
\end{aligned}
$$

$[j i ; b c d]_{\text {lin }}:$

$d_{l i}+\cdots=0$

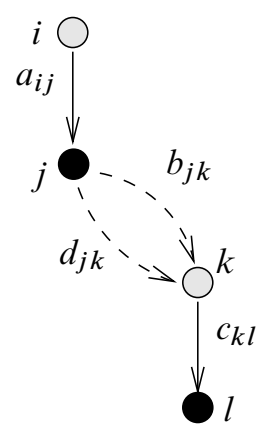

$[i l ; a b c]_{\operatorname{lin}}$ :

$$
b_{j k}+\cdots=0
$$

$[i l ; a d c]_{\operatorname{lin}}$ :

$d_{j k}+\cdots=0$

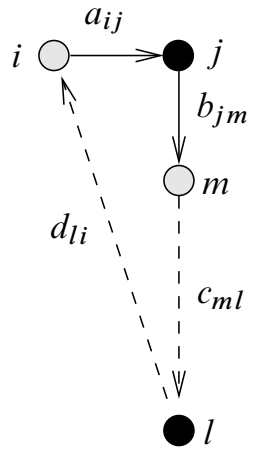

$[i l ; a b c]_{\operatorname{lin}}$ :

$c_{m l}+\cdots=0$

$[\operatorname{lm} ; d a b]_{\mathrm{lin}}:$

$d_{l i}+\cdots=0$

Figure 4: Configurations of arrows, and paired linear equations

I now claim that it is possible to pair these linearized equations so that the embedding $T_{\left[M_{\pi}\right]} X \hookrightarrow T_{\left[M_{\pi}\right]} S$ is cut out by an even number of linearly independent equations, proving the lemma. The proof proceeds by induction on the size of the pyramid partition $\pi$, the claim being obvious for small partitions.

Take a stone $l \in \pi$ so that $\pi \backslash\{l\}$ is also a partition. Assume first that the stone $l$ only touches one other stone of $\pi$. There is then only one nonzero incoming arrow $c_{k l}$ to $l$, and $l$ is not at the end of a chain of three consecutive nonzero arrows $a_{i j}, b_{j k}, c_{k l}$, since (by the relations) that would necessitate another nonzero incoming arrow $a_{n l}$. It is now easy to check that there are only three possible configurations of two nonzero 
arrows, leading to new nonzero linear relations, being part of a path involving $l$. The three configurations are depicted on Figure 4, together with the pairs of linear equations that the configurations give rise to. In the figure, solid arrows represent ones, whereas dotted arrows represent zeros. For example, in the middle case, we have two different nonzero arrows $a_{i j}, c_{k l}$, and there are two ways of completing the pair to a path: $a_{i j} b_{j k} c_{k l}$ and $a_{i j} d_{j k} c_{k l}$, leading to two linear relations cutting out the linear subspace of interest.

It is immediate that in all cases the linear equations paired are indeed linearly independent, and the pairs are well-defined. This proves the claim, and hence the lemma, in this case. The second case, where there are two nonzero incoming arrows $c_{k l}$ and $a_{n l}$ to $l$, is similar, with a slightly larger number of diagrams. The details are left to the reader.

Corollary 2.5.3 Let $\mathbf{v}=\left(d_{0}, d_{1}\right)$ be a dimension vector. The parity of the dimension of the Zariski tangent space at a $T_{W}$-fixed point $P \in\left(\mathcal{M}_{\mathbf{v}}\right)^{T_{W}}$ is the same as the parity of $d_{1}$.

Proof Let $Q \in X$ lie above $P \in \mathcal{M}_{\mathbf{v}}$. Since the $G$-action is free,

$$
\operatorname{dim} T_{P} \mathcal{M}_{\mathbf{v}}=\operatorname{dim} T_{Q} X-\operatorname{dim} G .
$$

On the other hand, using Lemma 2.5.2 and the fact that $S$ is just a vector space,

$$
\operatorname{dim} T_{Q} X \equiv \operatorname{dim} T_{Q} S=\operatorname{dim} S(\bmod 2) ;
$$

hence

$$
\operatorname{dim} T_{P} \mathcal{M}_{\mathbf{v}} \equiv \operatorname{dim} S-\operatorname{dim} G(\bmod 2) .
$$

Looking at the definitions of $S, G$, this difference of dimensions is indeed

$$
4 d_{0} d_{1}+d_{0}-d_{0}^{2}-d_{1}^{2} \equiv d_{1}(\bmod 2) \text {. }
$$

\subsection{Pyramid partitions and dimer configurations}

This section is not necessary for the logical flow of the paper. However, it points the way to generalizations, so I include it here.

Let $\mathcal{L} \cong \mathbb{Z}^{2}$ be the square lattice, thought of as an unoriented 4-regular bipartite graph with infinite vertex set $\mathcal{V}$ and edge set $\mathcal{E}$. Recall that a dimer configuration on the square lattice is a subset $\mathcal{F} \subset \mathcal{E}$ of the edges, so that every vertex $v \in \mathcal{V}$ is incident to exactly one edge in $\mathcal{F}$. Given a fixed dimer configuration $\mathcal{F}_{1}$, a dimer configuration is said to be asymptotic to $\mathcal{F}_{1}$, if the set $\mathcal{F} \backslash \mathcal{F}_{1}$ is finite.

Let $\mathcal{F}_{1}$ be the "length one empty room" square dimer configuration shown on Figure 5. 


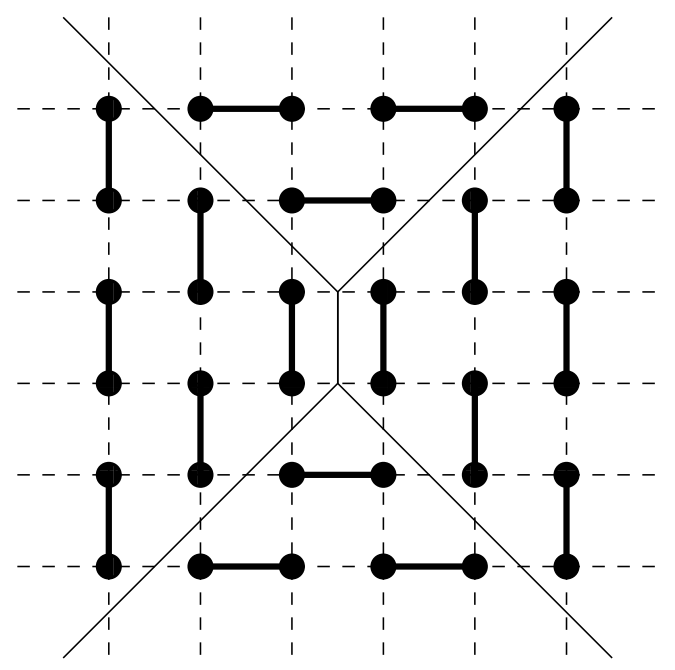

Figure 5: The "length one empty room" square dimer configuration $\mathcal{F}_{1}$

Proposition 2.6.1 There is a one-to-one correspondence between pyramid partitions $\alpha \in \mathcal{P}$ and dimer configurations $\mathcal{F}$ on the square lattice $\mathcal{L}$ asymptotic to the configuration $\mathcal{F}_{1}$.

Proof This is analogous to the correspondence between honeycomb dimers and ordinary 3-dimensional partitions. Given a pyramid partiton $\alpha \in \mathcal{P}$, consider its complement in the infinite pyramid arrangement. Now associate to this complement a dimer configuration a $\mathcal{F}$ as follows. Looking from above, impose a square grid in which the squares lie over the balls as on Figure 6. Now consider those edges which lie over balls or half-balls visible from above. As illustrated, black balls or half-balls correspond to horizontal dimers, whereas grey ones correspond to vertical dimers. The empty pyramid partition gives the "length one empty room" $\mathcal{F}_{1}$. Since $\alpha \in \mathcal{P}$ is finite, $\mathcal{F}$ is asymptotic to $\mathcal{F}_{1}$ and it is easy to see that all such arise.

Different pictures, perhaps more illuminating for some, can be found in Young [29, Figures 1 and 2].

Remark 2.6.2 A general periodic dimer model, under some extra conditions, also gives rise to a non-commutative toric algebra $A$ defined by a superpotential. Moreover, there is a correspondence between torus-fixed points in moduli spaces $\mathcal{M}_{k, \mathbf{v}}$ and dimer configurations with fixed asymptotic behaviour. This will be discussed in future work. 

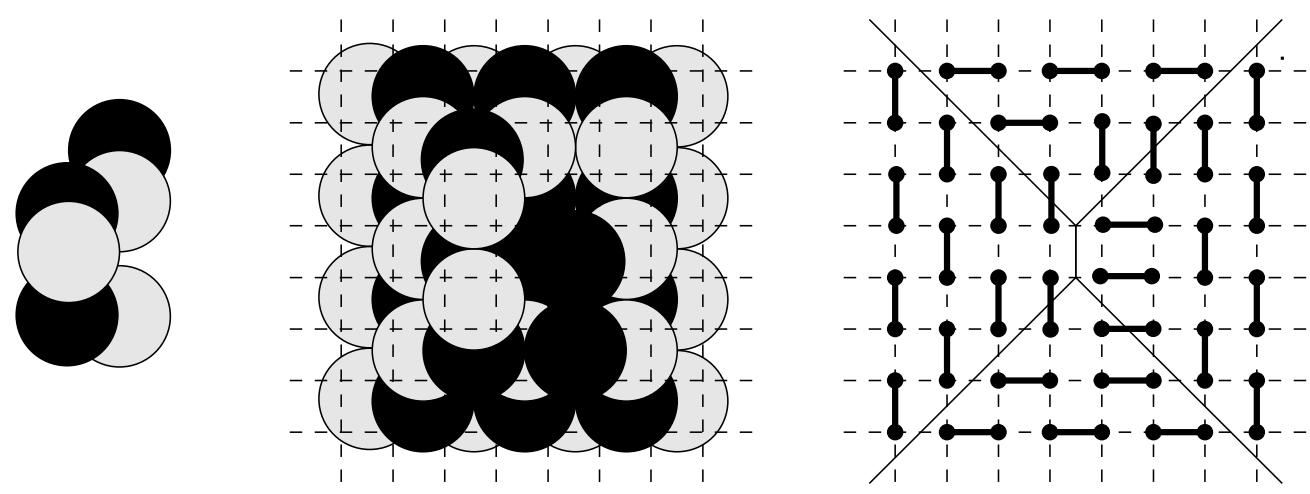

Figure 6: A pyramid partition, its complement with the grid, and the resulting dimer configuration

\subsection{The non-commutative partition function}

Consider the generating function

$$
Z_{A}(\mathbf{q})=\sum_{\mathbf{v} \in \mathbb{Z}^{2}} D_{\mathbf{v}} \mathbf{q}^{\mathbf{v}}
$$

of virtual counts of framed cyclic representations of $A$.

Theorem 2.7.1 The generating function $Z_{A}(\mathbf{q})$ can be expressed combinatorially as

$$
Z_{A}\left(q_{0}, q_{1}\right)=\sum_{\pi \in \mathcal{P}}(-1)^{\mathrm{wt}_{1}(\pi)} q_{0}^{\mathrm{wt}_{0}(\pi)} q_{1}^{\mathrm{wt}_{1}(\pi)}
$$

where $\mathcal{P}$ is the set of finite pyramid partitions, or equivalently by Proposition 2.6.1, the set of square dimer configurations $\mathcal{F}$ asymptotic to the "length one empty room" $\mathcal{F}_{1}$.

Proof The rank-2 torus $T_{W}$ acts on all moduli spaces $\mathcal{M}_{\mathbf{v}}$. By construction, the symmetric obstruction theory constructed in Theorem 1.3.1 is $T_{W}$-equivariant in the sense of [4]. Since $D_{\mathbf{v}}$ is a weighted Euler characteristic, with weights which are constant on $T_{W}$-orbits, nontrivial orbits do not contribute, and hence $D_{\mathbf{v}}$ receives contributions from torus-fixed points only. At a $T_{W}$-fixed point $P \in\left(\mathcal{M}_{\mathrm{v}}\right)^{T_{W}}$, the contribution is simply $(-1)^{\operatorname{dim} T_{P} \mathcal{M}_{\mathrm{v}}}$ by applying [4, Theorem 3.4] to a sufficiently general one-dimensional subtorus of $T_{W}$. Hence, the statement follows from Corollary 2.5.3.

The following result was conjectured in an earlier version of this paper, on the basis of extensive computational evidence, generalizing the one-variable specialization conjectured earlier by Kenyon [20]. 
Theorem 2.7.2 (Young [29]) The partition function $Z_{A}(\mathbf{q})$ admits the following infinite product expansion.

$$
Z_{A}\left(q_{0}, q_{1}\right)=M\left(-q_{0} q_{1}\right)^{2} \prod_{k \geq 1}\left(1+q_{0}^{k}\left(-q_{1}\right)^{k-1}\right)^{k}\left(1+q_{0}^{k}\left(-q_{1}\right)^{k+1}\right)^{k} .
$$

\section{Interpretations and generalizations}

\subsection{The commutative partition function}

Recall the rank one Donaldson-Thomas partition function [23] of the smooth commutative Calabi-Yau resolution $X$, the resolved conifold. The resolution $\pi: X \rightarrow Z$ contracts a single rational curve $C \subset X$ to the singular point of $Z$. Let $D_{n, d}$ be the DT invariant (Thomas [27]) of ideal sheaves $\mathcal{I}$ on $X$ with $\chi(\mathcal{I})=n$ and homology class $d[C]$. Define the DT partition function of $X$ as the series

$$
Z_{X}(q, t)=\sum_{n \in \mathbb{Z}} \sum_{d \geq 0} D_{d}^{n} q^{n} e^{-d t}
$$

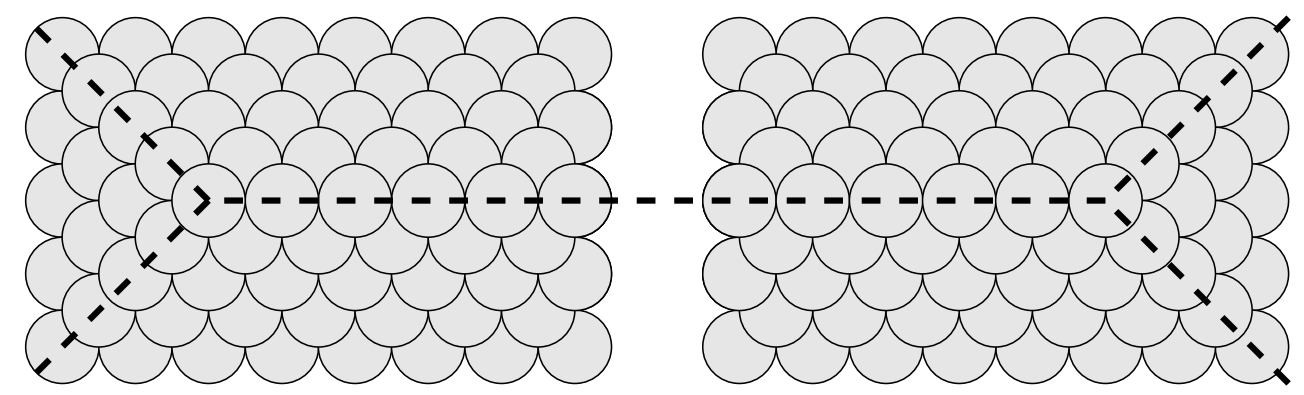

Figure 7: The combinatorial arrangement for the commutative resolution, with the toric web diagram indicated

An argument using torus localization, similar to the one given above, gives the following combinatorial interpretation of these DT invariants. Up to sign, $D_{d}^{n}$ is the number of pairs of semi-infinite 3-dimensional partitions $\left(\alpha_{1}, \alpha_{2}\right)$, bounded in the direction of two coordinate axes, with infinitely long necks in the third direction with a common ordinary partition $\lambda \vdash d$ as cross-section, and having total (renormalized) volume $\left|\alpha_{1}\right|+\left|\alpha_{2}\right|=n-f(\lambda)$, where $f(\lambda)$ is a certain combinatorial invariant [23, Lemma 5] of the partition $\lambda$. In other words, $D_{d}^{n}$ counts certain subsets of the arrangement of Figure 7; note that the shape of the latter is closely related to the toric combinatorics of the resolved conifold $X$ (indicated by the dashed line). Consequently [24; 18; 23], one obtains the following theorem. 


\section{Theorem 3.1.1}

$$
Z_{X}(q, t)=M(-q)^{2} \prod_{k \geq 1}\left(1-(-q)^{k} e^{-t}\right)^{k}
$$

In fact, the singularity $Z=\operatorname{Spec}(R)$ admits two, isomorphic, crepant resolutions $X, X^{+}$related by a flop $X^{+} \rightarrow X$. Under the natural isomorphism $H^{2}(X, \mathbb{R}) \rightarrow$ $H^{2}\left(X^{+}, \mathbb{R}\right)$ induced by the flop, the positive classes have opposite sign. Therefore the DT partition function of $X^{+}$can be written in the variables $q, t$ as

$$
Z_{X^{+}}(q, t)=M(-q)^{2} \prod_{k \geq 1}\left(1-(-q)^{k} e^{t}\right)^{k}
$$

\subsection{Partition functions as functions on Bridgeland space and wall cross- ing}

The formulae (3), (4) and (5) are closely related. The change of variables $q=q_{0} q_{1}$, $z=q_{1}=e^{-t}$ gives

$$
\begin{aligned}
Z_{X}(q, z) & =M(-q)^{2} \prod_{k \geq 1}\left(1-(-q)^{k} z\right)^{k}, \\
Z_{A}(q, z) & =M(-q)^{2} \prod_{k \geq 1}\left(1-(-q)^{k} z\right)^{k}\left(1-(-q)^{k} z^{-1}\right)^{k}, \\
Z_{X^{+}}(q, z) & =M(-q)^{2} \prod_{k \geq 1}\left(1-(-q)^{k} z^{-1}\right)^{k} .
\end{aligned}
$$

Hence dividing by the MacMahon factors, the reduced partition functions satisfy the curious formal factorization property

$$
Z_{A}^{\prime}(q, z)=Z_{X}^{\prime}(q, z) Z_{X^{+}}^{\prime}(q, z) .
$$

The variable $z=e^{-t}$ coordinatizes a parameter space naturally associated to the problem. To discuss this space, I need some definitions. Recall the map $\pi: X \rightarrow Z$, contracting the rational curve $C$. Let $\mathcal{D}(X / Z)$ denote the bounded derived category of coherent sheaves on $X$, supported on a neighbourhood of the exceptional curve $C$. It is known [6] that $\mathcal{D}(X / Z)$ is equivalent to the derived category $\mathcal{D}_{\text {nilp }}(A-\operatorname{Mod})$ of complexes of finitely generated $A$-modules with locally nilpotent cohomology modules.

Let $\operatorname{Stab}(X / Z)$ denote the component of the space of normalized stability conditions (Bridgeland [9; 8]) on the category $\mathcal{D}(X / Z)$, which contains stability conditions whose heart is the category of sheaves on $X$ supported along $C$. By [9], $\operatorname{Stab}(X / Z)$ is a 
one-dimensional complex manifold. Let finally $\operatorname{DAut}(X / Z)$ be the subgroup of the derived autoequivalence group of $\mathcal{D}(X / Z)$ which preserves this component. Then it is proved in Bridgeland [8] and Toda [28] that

$$
\operatorname{Stab}(X / Z) / \operatorname{DAut}(X / Z) \cong \mathbb{P}^{1} \backslash\{0,1, \infty\} .
$$

In physics language, this is the full complexified Kähler structure moduli space of $X$.

The space $\operatorname{Stab}(X / Z) / \operatorname{DAut}(X / Z)$ and its structures are pictured on Figure 8. The parameter $t$ naturally lives in the upper or lower half planes $\{B+i \omega \mid \pm \omega>0\}$ of the complexified cohomology space $H^{2}(X, \mathbb{C}) \cong \mathbb{C}$, with $\omega=-i \operatorname{Im}(t)$ measuring the volume of the embedded projective line in $X$. After taking the quotient by $\operatorname{DAut}(X / Z)$, $e^{-t}$ lives in the punctured upper and lower hemispheres in $\mathbb{P}^{1} \backslash\{0,1, \infty\}$. The two cusps $z=0, \infty$ correspond to the "large volume" limits $t \rightarrow \pm \infty$ of $X, X^{+}$. For a stability condition corresponding to a point on the upper or lower hemispheres $\{|z|>1\},\{|z|<1\}$, the $t$-structure on $\mathcal{D}(X / Z)$ has heart $\operatorname{Coh}(X / Z)$, respectively $\operatorname{Coh}\left(X^{+} / Z\right)$ (up to shift). Along the punctured equator $\{|z|=1\} \backslash\{z=1\}$, all stability conditions correspond to the perverse $t$-structure on $\mathcal{D}(X / Z)$, with heart $A$-nilp, the category of nilpotent $A$-modules. In this sense, the cusp $z=1$ is naturally associated to the non-commutative algebra $A$.

At this point, one would like to say that the functions $Z_{A}, Z_{X}, Z_{X^{+}}$count objects from $\mathcal{D}(X / Z)$, which are "stable in the limit". This is however not correct as it stands. The commutative DT partition function $Z_{X}$ of $X$ certainly counts objects of $\operatorname{Coh}(X / Z)$, at least if one thinks of it as counting structure sheaves rather than ideal sheaves. However, most of these are not stable: for example, the sheaf $E=\mathcal{O}_{X} / \mathcal{I}_{C}^{2}$ on $C$, where $\mathcal{I}_{C}$ is the ideal defining the curve $C$, sits in a sequence

$$
0 \rightarrow \mathcal{O}_{C}(1)^{\oplus 2} \rightarrow E \rightarrow \mathcal{O}_{C} \rightarrow 0
$$

which is a destabilizing subsequence for any stability condition on $\mathcal{D}(X / Z)$ with heart $\operatorname{Coh}(X / Z)$. Similarly, $Z_{A}$ does count objects of $A$-nilp, since all $T$-fixed $A-$ representations are in fact nilpotent, but most of these are non-stable (though semistable) in $\mathcal{D}_{\text {nilp }}(A-$ mod $)$.

An alternative is to return to the original interpretation of the DT partition function $Z_{X}$ of $X$ as a generating function counting ideal sheaves. These are indeed Gieseker stable on $X$, but they are not objects of $\mathcal{D}(X / Z)$, only of $\mathcal{D}(X)$. However, very little is known about the space of (normalized) stability conditions on $\mathcal{D}(X)$, though presumably it is also a cover of the thrice punctured Riemann sphere. It is conceivable that near the large volume limit point corresponding to $X$, every Gieseker stable sheaf on $X$ becomes Bridgeland stable, so $Z_{X}$ does in fact count stable objects near a cusp 
in the space of stability conditions. Similarly, by Proposition 1.4.1, the function $Z_{A}$ can be thought of as counting certain finite-codimension ideals in $A$, and one might hope that in fact these, and only these, objects of $\mathcal{D}(X) \cong \mathcal{D}(A-$ Mod $)$ are stable under an appropriate limiting stability condition as one approaches the conifold point $z=1$ along the equator. Note that there are two ways to do that, perhaps corresponding to the two choices of vertex for the cyclic generator. (Compare Denef-Moore [11, Section 6.1.2] for a similar example of "opposite" sets of objects becoming stable along two opposite rays leading to the same limit point). The change from $Z_{X}$ to $Z_{A}$ to $Z_{X^{+}}$might then be interpreted as an instance of wall crossing in the space of stability conditions, with a countably infinite number of walls between $z=0$ and $z=1$, respectively $z=1$ and $z=\infty$. It would be very interesting to pursue this direction further.

Note finally that although $Z_{A}$ has a Taylor series expansion in the variables $\left(q_{0}, q_{1}\right)=$ $(q / z, z)$, it is only a Laurent series in the variables $(q, z)$ near $z=0$ or $z=\infty$ with infinitely many positive and negative powers of $z$. In this sense, this change of variables is "non-perturbative". As in (7), $Z_{X}$ and $Z_{X^{+}}$are the positive and negative parts of $Z_{A}$ under a factorization on $\mathbb{P}_{z}^{1}$, reminiscent of Birkhoff factorization; these can then be expanded as Taylor series near the appropriate cusps.

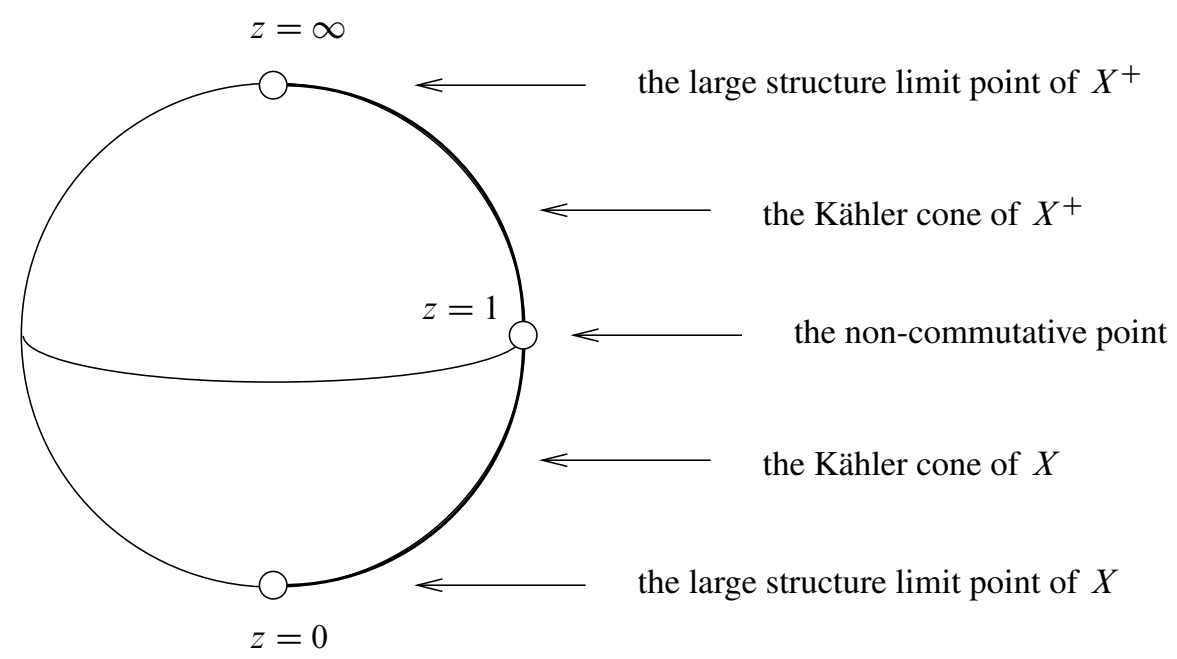

Figure 8: The Kähler structure moduli space of $X$ 


\subsection{Coefficients at small volume and "modularity"}

Starting with the form (6) of the partition function $Z_{A}$, under the substitution $z=e^{-t}$ discussed in the last section, write

$$
\begin{aligned}
\log Z_{A}(q, t) & =\sum_{k \geq 1} k \cdot\left(\log \left(1-(-q)^{k} e^{-t}\right)+\log \left(1-(-q)^{k} e^{t}\right)-2 \log \left(1-(-q)^{k}\right)\right) \\
& =\sum_{k, n \geq 1} \frac{k}{n}(-q)^{k n}\left(e^{-n t}+e^{n t}-2\right) \\
& =\sum_{d \geq 1} d \cdot(-q)^{d} \cdot \sum_{n \mid d} \frac{1}{n^{2}}\left(2 \sinh \frac{n t}{2}\right)^{2} .
\end{aligned}
$$

This is formally very similar to the way the change of variables $q=-e^{i u}$ of [23] leads to the Gopakumar-Vafa form [15] of the closed string partition function. However, it is very different in substance, since $t$ is the Kähler parameter. Thus (8) is valid near small volume, or equivalently high spacetime curvature, and large string coupling $u$. Note that it is essential to start with the whole $Z_{A}$, including its positive and negative parts as well as the MacMahon factor, to get this form.

To continue, use the series expansion

$$
\left(2 \sinh \frac{x}{2}\right)^{2}=\sum_{k \geq 1} \frac{2}{(2 k) !} x^{2 k}
$$

to get

$$
\begin{aligned}
\log Z_{A}(q, t) & =\sum_{d, k \geq 1} \frac{2 d}{(2 k) !}(-q)^{d} t^{2 k} \cdot \sum_{n \mid d} n^{2 k-2} \\
& =\sum_{d, k \geq 1} \frac{2(-1)^{d} d \sigma_{2 k-2}(d)}{(2 k) !} q^{d} t^{2 k},
\end{aligned}
$$

with the divisor power sum function for positive integers $d$ defined by

$$
\sigma_{s}(d)=\sum_{n \mid d} n^{s}
$$

The expression (9) has the form of a generating series for a set of new "(connected) enumerative invariants"

$$
M_{d, k}=\frac{2(-1)^{d} d \cdot \sigma_{2 k-2}(d)}{(2 k) !}, \quad d, k \geq 1,
$$


associated to $X$, at small volume/high curvature and large string coupling. I have no geometric or physical interpretation for these numbers at present.

To continue, for integers $l \geq 1$, introduce the series

$$
E_{l}(x)=\sum_{d \geq 1} \sigma_{l-1}(d) x^{d} .
$$

The reason for the notation will become apparent presently. (9) becomes

$$
\log Z_{A}(q, t)=\sum_{k \geq 1} \frac{2}{(2 k) !} t^{2 k} q \frac{d}{d q} E_{2 k-1}(-q) .
$$

On the other hand, the standard identity (valid for $\operatorname{Im} \tau>0, l>1$ )

$$
\sum_{n \in \mathbb{Z}} \frac{1}{(\tau+n)^{l}}=\frac{(-2 \pi i)^{l}}{(l-1) !} \sum_{m \geq 1} m^{l-1} e^{2 \pi i m \tau},
$$

leads for $l \geq 2$ to

$$
E_{l}\left(e^{2 \pi i \tau}\right)=\frac{(l-1) !}{(-2 \pi i)^{l}} \sum_{m \geq 1, n \in \mathbb{Z}} \frac{1}{(m \tau+n)^{l}} .
$$

Thus, $E_{l}$ is "essentially" an Eisenstein series. Indeed, for $l=2 k$ even, we can double the sum to run over $m \in \mathbb{Z} \backslash\{0\}, n \in \mathbb{Z}$ and add the appropriate constant term, to get the standard modular (quasi-)invariant Eisenstein series $G_{2 k}$. However, for the odd values of interest in (10), the signs work against us. So $E_{2 k-1}$ is not modular, but it is intriguingly close to being so. Compare Dabholkar et al [10, Appendix E] for a similar discussion involving the MacMahon function.

\subsection{A generalized partition function}

The combinatorial arragement of Figure 2, leading to pyramid partitions, has an obvious generalization to an elongated rectangular pyramid with $n$ black stones on level 0 . In the dimer model, finite subsets of this arrangement correspond to configurations with the "length $n$ empty room" asymptotics.

Let $\mathcal{P}^{(n)}$ denote the set of finite partition-like subsets of the elongated pyramid configuration of length $n$; as before, let $\mathrm{wt}_{i}(\pi)$ denote the number of stones in $\alpha \in \mathcal{P}^{(n)}$ of colour $i$. Define the partition function

$$
Z_{A}^{(n)}\left(q_{0}, q_{1}\right)=\sum_{\pi \in \mathcal{P}^{(n)}} q_{0}^{\mathrm{wt}_{0}(\pi)}\left(-q_{1}\right)^{\mathrm{wt}_{1}(\pi)} .
$$


The choice of signs is motivated by Corollary 2.5.3, but this is only by analogy, since the precise enumerative interpretation of these invariants is unclear. The following generalization of Theorem 2.7.2 was again conjectured in an earlier version of this paper (compare Kenyon [20] for the one-variable specialization), based on computational evidence.

Theorem 3.4.1 (Young [29]) The partition function $Z_{A}^{(n)}(\mathbf{q})$ admits the following infinite product expansion.

$$
\begin{gathered}
Z_{A}^{(n)}\left(q_{0}, q_{1}\right)= \\
M\left(-q_{0} q_{1}\right)^{2} \prod_{k \geq 1}\left(1+q_{0}^{k}\left(-q_{1}\right)^{k-1}\right)^{k+n-1} \\
\left(1+q_{0}^{k}\left(-q_{1}\right)^{k+1}\right)^{\max (k-n+1,0)}
\end{gathered}
$$

In the recent physics literature on the conifold, there are expressions which are remarkably similar to the terms appearing as the ratio $Z_{A}^{(n)} / Z_{A}$; compare Hyun-Yi [17, (3.30)-(3.32)] as well as Kashani-Poor [19, (3.6)]. These computations are in the context of Lagrangian branes, so not immediately applicable, but they suggest a relationship between these generalized partition functions and higher-rank DonaldsonThomas theory. I hope to return to this point in future work.

\subsection{The global case}

In this section, I indicate a possible extension of the ideas of the paper to a global context. Assume that $Y$ is a projective variety, singular at a finite set of conifold points (nodes). Assume further that there is a Calabi-Yau small resolution $\pi: X \rightarrow Y$, together with a line bundle $\mathcal{L} \in \operatorname{Pic}(X)$ such that $\left.\mathcal{L}\right|_{C_{i}} \cong \mathcal{O}_{C_{i}}(1)$ on all $\pi$-exceptional curves $C_{i} \cong \mathbb{P}^{1}$.

Proposition 3.5.1 (Van den Bergh) There exists a sheaf $\mathcal{A}$ of associative, noncommutative algebras on $Y$, such that over $Y \backslash \operatorname{Sing}(Y), \mathcal{A}_{Y \backslash \operatorname{Sing}(Y)}$ is a sheaf of Azumaya algebras (locally, matrix algebras over the structure sheaf), and in a neighbourhood of each of the nodes, sections of $\mathcal{A}$ define the non-commutative resolution $A$ discussed before. Further, there is a derived equivalence $\mathcal{D}(Y) \cong \mathcal{D}(\mathcal{A}-$ mod $)$.

Proof Define the sheaf $\mathcal{A}$ of $\mathcal{O}_{Y}$-algebras by

$$
\mathcal{A}=\pi_{*} \operatorname{End}_{X}\left(\mathcal{O}_{X} \oplus \mathcal{L}\right) .
$$

This is clearly a sheaf of Azumaya algebras on $Y \backslash \operatorname{Sing}(X)$, whereas by [6], in a neighbourhood of each of the nodes, sections of $\mathcal{A}$ define the noncommutative crepant resolution $A$. The derived equivalence follows from [6, Propositions 3.3.1-3.3.2]. 
Thus, $\mathcal{D}(\mathcal{A}-$ mod $)$ is a $3-$ Calabi-Yau category, with Serre functor the third power of the shift functor, and thus appropriately defined moduli spaces of (stable) $\mathcal{A}$-modules will carry a perfect obstruction theory. It would be interesting to compare the invariants thus obtained to the ordinary DT invariants of $Y$.

\subsection{Some quotient examples}

Phenomena similar to the above have been observed by Bryan-Young [30] for some orbifolds $\mathbb{C}^{3} / \Gamma$, where the singularity admits a semismall resolution (no divisors over points, equivalently all exceptional fibres are rational curves), such as $\Gamma<\operatorname{SL}(2, \mathbb{C})<$ $\operatorname{SL}(3, \mathbb{C})$ and $\Gamma=\mathbb{Z} / 2 \times \mathbb{Z} / 2<\operatorname{SL}(3, \mathbb{C})$. Let $X \rightarrow \mathbb{C}^{3} / \Gamma$ be the canonical crepant resolution given by the $\Gamma$-Hilbert scheme. Consider also the canonical non-commutative resolution given by the cross product algebra

$$
A=\mathbb{C}\left[x_{1}, x_{2}, x_{3}\right] \star \mathbb{C} \Gamma .
$$

This algebra is known to be (Morita equivalent to) the quiver algebra of the McKay quiver of $\Gamma$, with a specific superpotential, see Ginzburg [14, Theorem 4.4.6].

In complete analogy with the above discussion, there is a commutative DonaldsonThomas partition function $Z_{X}$, as well as a noncommutative partition function $Z_{A}$. Assuming further that $\Gamma$ is abelian, both can again be computed by torus localization; the computation of $Z_{A}$ localizes to a set indexed by standard 3-dimensional partitions coloured with characters of the group $\Gamma$.

The resulting formulae depend on a set of roots $\Sigma \subset \mathbb{Z}^{r}$, together with a subset $\Sigma^{+} \subset \Sigma$ of positive roots. For a set of dual variables $\mathbf{t}$, the commutative partition function takes the form

$$
Z_{X}(q, \mathbf{t})=M(-q)^{\chi} \prod_{k \geq 1} \prod_{\alpha \in \Sigma^{+}}\left(1-(-q)^{k} e^{-\langle\alpha, \mathbf{t}\rangle}\right)^{ \pm k}
$$

(product over positive roots), whereas $Z_{A}$ is of the form

$$
Z_{A}(q, \mathbf{t})=M(-q)^{\chi} \prod_{k \geq 1} \prod_{\alpha \in \Sigma}\left(1-(-q)^{k} e^{-\langle\alpha, \mathbf{t}\rangle}\right)^{ \pm k}
$$

(product over all roots). In further analogy with the case of the resolved conifold, the resolution $X$ admits various flops $X^{i}$ (geometric or derived) which have partition functions $Z_{X^{i}}$ obtained by choosing a different Weyl chamber as dictated by the flop. In particular, there is a flop $X^{+}$of $X$ corresponding to the opposite chamber, together with a factorization

$$
Z_{A}^{\prime}(q, \mathbf{t})=Z_{X}^{\prime}(q, \mathbf{t}) Z_{X^{+}}^{\prime}(q, \mathbf{t})
$$

as in (7) above. 
Acknowledgements I would like to thank Aravind Asok, Kai Behrend, Tom Bridgeland, Jim Bryan, Alastair Craw, Victor Ginzburg, Dominic Joyce, Richard Kenyon, Raphaël Rouquier, Ed Segal, Yukinobu Toda and Ben Young for helpful comments and correspondence, and the referee for his careful reading of the manuscript. Special thanks are due to Alastair King for introducing me to quiver algebras and answering several questions; the main idea of the paper was formulated after a conversation with him. My research was partially supported by OTKA grant K61116. The final version of the paper was prepared while I enjoyed the hospitality of the Rényi Institute of Mathematics in Budapest, Hungary, supported by the EU BudAlgGeo project.

\section{References}

[1] M Artin, J J Zhang, Abstract Hilbert schemes, Algebr. Represent. Theory 4 (2001) 305-394 MR1863391

[2] K Behrend, Donaldson-Thomas invariants via microlocal geometry (2005) arXiv: math/0507523

[3] K Behrend, B Fantechi, The intrinsic normal cone, Invent. Math. 128 (1997) 45-88 MR1437495

[4] K Behrend, B Fantechi, Symmetric obstruction theories and Hilbert schemes of points on threefolds (2005) arXiv:math/0512556

[5] M van den Bergh, Non-commutative crepant resolutions, from: "The legacy of Niels Henrik Abel”, Springer, Berlin (2004) 749-770 MR2077594

[6] M van den Bergh, Three-dimensional flops and noncommutative rings, Duke Math. J. 122 (2004) 423-455 MR2057015

[7] R Bocklandt, Graded Calabi Yau algebras of dimension 3, J. Pure Appl. Algebra 212 (2008) 14-32 MR2355031

[8] T Bridgeland, Derived categories of coherent sheaves, from: "International Congress of Mathematicians. Vol. II”, Eur. Math. Soc., Zürich (2006) 563-582 MR2275610

[9] T Bridgeland, Stability conditions on triangulated categories, Ann. Math. 166 (2007) $317-345$

[10] A Dabholkar, F Denef, G W Moore, B Pioline, Precision counting of small black holes, J. High Energy Phys. (2005) 096, 90 pp. MR2180982

[11] F Denef, G W Moore, Split states, entropy enigmas, holes and halos (2007) arXiv: hep-th/0702146

[12] B Feng, Y-H He, K D Kennaway, C Vafa, Dimer models from mirror symmetry and quivering amoebae (2005) arXiv:hep-th/0511287 
[13] S Franco, A Hanany, D Vegh, B Wecht, KD Kennaway, Brane dimers and quiver gauge theories, J. High Energy Phys. (2006) 096, 48 pp. MR2201227

[14] V Ginzburg, Calabi-Yau algebras (2006) arXiv:math/0612139

[15] R Gopakumar, C Vafa, M-theory and topological strings (1998) arXiv: hep-th/9809187

[16] A Hanany, KD Kennaway, Dimer models and toric diagrams (2005) arXiv: hep-th/0503149

[17] S Hyun, S-H Yi, Non-compact topological branes on conifold, J. High Energy Phys. (2006) 075, 34 pp. MR2270388

[18] A Iqbal, N Nekrasov, A Okounkov, C Vafa, Quantum foam and topological strings (2003) arXiv:hep-th/0312022

[19] A-K Kashani-Poor, The wave function behavior of the open topological string partition function on the conifold, J. High Energy Phys. (2007) 004, 47 pp. MR2318726

[20] R Kenyon, Talk at the Workshop on random partitions and Calabi-Yau crystals, Amsterdam (2005) http: //www. math. brown.edu/ rkenyon/talks/pyramids . pdf

[21] A D King, Moduli of representations of finite-dimensional algebras, Quart. J. Math. Oxford Ser. (2) 45 (1994) 515-530 MR1315461

[22] I R Klebanov, E Witten, Superconformal field theory on threebranes at a Calabi-Yau singularity, Nuclear Phys. B 536 (1999) 199-218 MR1666725

[23] D Maulik, N Nekrasov, A Okounkov, R Pandharipande, Gromov-Witten theory and Donaldson-Thomas theory. I, Compos. Math. 142 (2006) 1263-1285 MR2264664

[24] A Okounkov, N Reshetikhin, C Vafa, Quantum Calabi-Yau and classical crystals, from: "The unity of mathematics", Progr. Math. 244, Birkhäuser, Boston (2006) 597618 MR2181817

[25] E Segal, The A-infinity deformation theory of a point and the derived categories of local Calabi-Yaus (2007) arXiv:math/0702539

[26] R P Thomas, Gauge theory on Calabi-Yau manifolds, DPhil Thesis, University of Oxford (1997)

[27] R P Thomas, A holomorphic Casson invariant for Calabi-Yau 3-folds, and bundles on K3 fibrations, J. Differential Geom. 54 (2000) 367-438 MR1818182

[28] Y Toda, Stability conditions and crepant small resolutions (2005) arXiv: math/0512648

[29] B Young, Computing a pyramid partition generating function with dimer shuffling (2007) arXiv:0709.3079

[30] B Young, Generating functions for colored 3D Young diagrams and the DonaldsonThomas invariants of orbifolds (2008) arXiv:0802.3948 With an appendix by J Bryan 
Mathematical Institute, University of Oxford

24-29 St Giles', Oxford, OX1 3LB, UK

szendroi@maths.ox.ac.uk

http://www . maths.ox.ac.uk/ szendroi

Proposed: Jim Bryan

Received: 24 July 2007

Seconded: Lothar Goettsche, Simon Donaldson

Revised: 9 January 2008

Geometry ${ }^{3}$ Topology, Volume 12 (2008) 